\title{
ConsenSur II: Origen y misión
}

Carlos Bantar

\author{
Consensur II: Origin and mission
}

\section{$\mathrm{L}$}

a neumonía adquirida en la comunidad (NAC) en adultos es, probablemente, una de las infecciones que afecta a pacientes ambulatorios para la cual se ha escrito la mayor diversidad de guías en todo el mundo. Si bien la mayoría de ellas coinciden en que la terapia antimicrobiana debería seleccionarse sobre parámetros como la gravedad de la enfermedad, la presencia de comorbilidades y la epidemiología, se puede apreciar una dispersión considerable en la elección de las opciones de primera línea entre los diferentes países, aun en los casos considerados de bajo riesgo. Aunque las diferentes guías pueden ser útiles para lograr el manejo apropiado de la NAC, cada una de ellas tiene sus propias ventajas y limitaciones. Aun así, algunos países o regiones adoptan otras guías o diseñan las propias sin tener en cuenta los datos locales, probablemente debido a la escasa disponibilidad de éstos. Sin embargo, tanto la epidemiología de la NAC como las características de los sistemas de salud pueden presentar diferencias entre distintas regiones. A mediados de año 2001 se constituyó un grupo de trabajo integrado por infectólogos y microbiólogos del Cono Sur americano con evidente interés y experiencia personal en NAC en adultos. Dicho grupo (ConsenSur) tuvo como objetivo diseñar una guía práctica para el manejo inicial de esta patología, concebida sobre datos locales. El documento resultante se publicó a fines de 2002. Sin embargo, varios temas merecen discutirse nuevamente, como sigue: ciertos puntajes clínicos además del Índice Fisiológico de Seve- ridad se hicieron más populares en la práctica clínica (por ej. CURB-65, CRB-65); emergieron algunos patógenos en la región, tales como Staphylococcus aureus resistente a meticilina adquirido en la comunidad (SARM-AC) y Legionella spp; se reportaron nuevas evidencias sobre el desempeño de la prueba rápida para el diagnóstico etiológico de NAC (por ejemplo, antígeno de Legionella en orina y antígeno de Streptococcus pneumoniae), deben abordarse nuevas consideraciones terapéuticas (por ej, reformulación de la dosis, duración del tratamiento, emergencia de nuevos antimicrobianos e impacto clínico del tratamiento combinado). Por tal motivo, se constituyó un grupo de trabajo, integrado por varios miembros del ConsenSur I, pero con el agregado de un nuevo país, Colombia, y de especialistas en neumología, que se suman a los microbiólogos clínicos e infectólogos en esta segunda versión, llamada ConsenSur II. El lector encontrará en esta entrega una propuesta de abordaje de la NAC en adultos, con características prácticas, desde el punto de vista clínico, que incluye la categorización sencilla de la gravedad del enfermo, la consecuente decisión del sitio de cuidado, la elección costo/efectiva de estudios adicionales y la selección razonable de la terapia antimicrobiana. Todos estos aspectos están basados en una profunda discusión realizada sobre las bases de las evidencias locales, analizadas y en muchos casos generadas, por los miembros del ConsenSur II, con experiencias propias evidenciables en la literatura científica.

Carlos Bantar Hospital San Martín

Paraná, Entre Rios, Argentina cbantar@arnet.com.ar 